\title{
Failure-experiment-supported optimization of poorly-reproducible synthetic conditions for novel lanthanide metal-organic
}

\section{frameworks}

\author{
Yu Kitamura, ${ }^{[a]}$ Emi Terado, ${ }^{[a]}$ Zechen Zhang, ${ }^{[b]}$ Hirofumi Yoshikawa, ${ }^{[b]}$ Tomoko Inose, ${ }^{[c, d]}$ Hiroshi Uji- \\ $\mathrm{i},{ }^{[c, e]}$ Masaharu Tanimizu, ${ }^{[f]}$ Akihiro Inokuchi, ${ }^{[g]}$ Yoshinobu Kamakura, ${ }^{\left[{ }^{[a]}\right.}$ and Daisuke Tanaka ${ }^{*[a, h]}$ \\ [a] Y. Kitamura, E. Terado, Y. Kamakura, Prof. D. Tanaka \\ Department of Chemistry, School of Science and Technology, \\ Kwansei Gakuin University \\ 2-1 Gakuen, Sanda, Hyogo 669-1337 (Japan) \\ E-mail: dtanaka@kwansei.ac.jp \\ [b] Z. Zhang, Prof. H. Yoshikawa \\ Department of Nanotechnology for Sustainable Energy, School of Science and Technology, \\ Kwansei Gakuin University \\ 2-1 Gakuen, Sanda, Hyogo 669-1337 (Japan) \\ [c] Dr. T. Inose, Prof. H. Uji-i \\ Division of Photonics and Optical Science, Research Institute for Electronic Science (RIES), \\ Hokkaido University \\ North 20 West 10, Kita ward, Sapporo, Hokkaido 001-0020 (Japan) \\ [d] Dr. T. Inose \\ Institute for Integrated Cell-Material Sciences (WPI-iCeMS), \\ Kyoto University \\ Yoshida, Sakyo-ku, Kyoto 606-8501 (Japan) \\ [e] Prof. H. Uji-i \\ Department of Chemistry, \\ Katholieke Universiteit Leuven, Celestijnenlaan 200F, Heverlee, 3001 (Belgium) \\ [f] Prof. M. Tanimizu \\ Department of Applied Chemistry for Environment, School of Science and Technology, \\ Kwansei Gakuin University \\ 2-1 Gakuen, Sanda, Hyogo 669-1337 (Japan) \\ [g] Prof. A. Inokuchi \\ Department of Informatics, School of Science and Technology, \\ Kwansei Gakuin University \\ 2-1 Gakuen, Sanda, Hyogo 669-1337 (Japan) \\ [h] Prof. D. Tanaka \\ JST PRESTO \\ 2-1 Gakuen, Sanda, Hyogo 669-1337 (Japan) \\ Supporting information for this article is given via a link at the end of the document.
}

\begin{abstract}
A series of novel metal organic frameworks with lanthanide double-layer-based inorganic subnetworks (KGF-3) was synthesized assisted by machine learning. Pure KGF-3 was difficult to isolate in the initial screening experiments. The synthetic conditions were successfully optimized by extracting the dominant factors for KGF-3 synthesis using two machine-learning techniques. Cluster analysis was used to classify the obtained PXRD patterns of the products and to decide automatically whether the experiments were successful or had failed. Decision tree analysis was used to visualize the experimental results, with the factors that mainly affected the synthetic reproducibility being extracted. The water adsorption isotherm revealed that KGF-3 possesses unique hydrophilic pores, and impedance measurements demonstrated good proton conductivities $\left(\sigma=5.2 \times 10^{-4} \mathrm{~S} \mathrm{~cm}^{-1}\right.$ for KGF-3(Y)) at a high temperature $(363 \mathrm{~K})$ and high relative humidity $(95 \%)$.
\end{abstract}

Lanthanide-based metal-organic frameworks (Ln-MOFs), which consist of lanthanide ions or clusters linked by organic linkers, are promising materials for a wide range of applications. ${ }^{[1]}$ Lanthanides have been reported to form giant clusters ${ }^{[2]}$ hence, multiple or novel properties are expected to arise due to synergism when such large clusters or more highly dimensional infinite structures are incorporated into the skeletons of MOFs. ${ }^{[3]}$ However, since the formation of lanthanide clusters is easily affected by the reaction conditions, rationally designing and synthesizing MOFs with Ln-cluster-based subnetworks is challenging. In addition, Ln-MOFs are known to provide many crystal polymorphisms due to their flexible coordination nature Ln-MOFs are generally synthesized using the solvothermal method in sealed reaction vessels; therefore, the understanding of the MOF crystallization process has been limited, which renders it difficult to evaluate the factors that influence the reaction. ${ }^{[4]}$ Therefore, the preparation of Ln-MOFs frequently suffers from a poor reproducibility.

Recently, machine learning has attracted attention as an efficient exploration tool, especially in the area of materials synthesis. ${ }^{[5]}$ Because machine learning can deal with synthetic results statistically, meaningful information can be extracted even for experiments that are poorly reproducible, thereby enabling us to understand the link between reaction conditions and synthetic results. However, the use of machine learning as a search tool for crystallization conditions remains limited ${ }^{[6]}$ and, to the best of our 


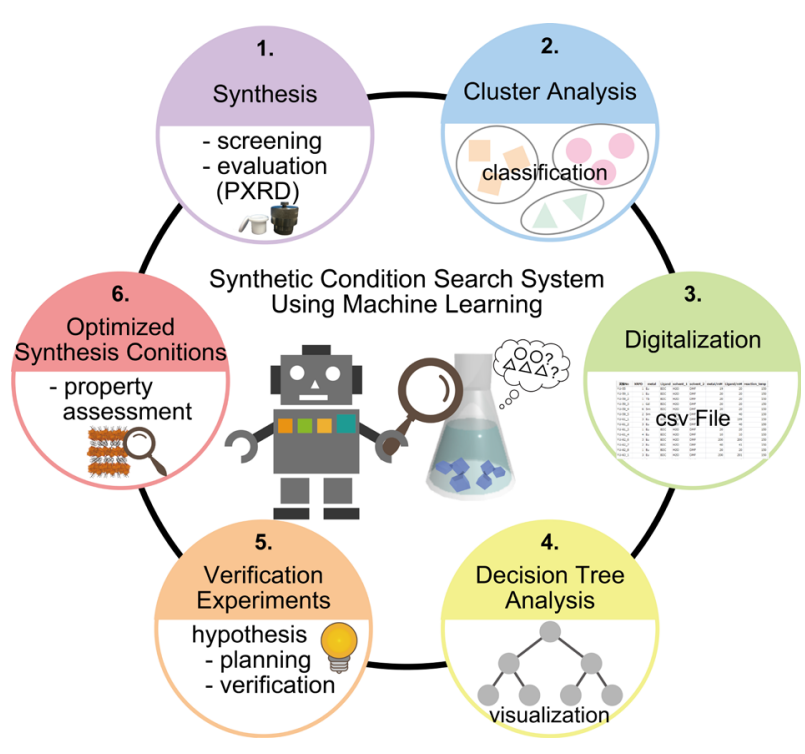

Figure 1. Schematic representation of the research flow process used to optimize the synthetic conditions incorporating machine-learning methods.

knowledge, no studies have introduced machine learning when exploring novel MOFs.

With this background in mind, we herein report the synthesis of a novel Ln-MOF with lanthanide-double-layer-based subnetworks (KGF-3) assisted by machine learning. The synthetic results are evaluated using both cluster analysis and decision tree analysis to interpret the experimental results. These analyses enabled us to realize the conditions for the reproducible synthesis of KGF-3. Figure 1 shows the flow process used to optimize the synthetic conditions using machine-learning techniques. Water adsorption experiments and impedance measurements were also used to analyze the pores and proton conductivity of the prepared KGF-3.

Solvothermal synthesis was performed using nitrate hexahydrate salts of lanthanide metals $\left(\mathrm{Ln}^{3+}=\mathrm{Sm}, \mathrm{Eu}, \mathrm{Gd}\right.$, and $\left.\mathrm{Tb}\right)$ in the presence of terephthalic acid (BDC) in $\mathrm{H}_{2} \mathrm{O} / \mathrm{DMF}$ (DMF $=N, N$-dimethylformamide). A total of 108 experiments were carried out, with variables including the lanthanide metal, the concentration of the metal and/or ligand solution, the reaction temperature and time, cooling time, and the type of reaction vessel (see Synthetic Conditions 1 in the SI). Solid powders were obtained under all conditions, and were characterized by powder $X$-ray diffractometry (PXRD) where, in some cases, novel phases were observed.

The novel $\left[\mathrm{Ln}_{10}(\mathrm{BDC})_{3}(\mathrm{HCOO})_{4}\left(\mu_{3}-\mathrm{OH}\right)_{12}\left(\mu_{5}-\mathrm{CO}_{3}\right)_{4}\left(\mathrm{H}_{2} \mathrm{O}\right)_{2}\right]$ phase, which we refer to as "KGF-3", was obtained in the presence of various lanthanide ions, although single crystals suitable for crystal structure analysis were obtained only when $\mathrm{Gd}^{3+}$ was used as the metal source. Based on the single-crystal X-ray data, KGF-3(Gd) was found to contain five types of nonequivalent $\mathrm{Gd}^{3+}$ ions with coordination numbers of eight or nine. Four complexes share ridges with each other to form a chain, which is then connected by another lanthanide ion to form a porous layer (Figure 2a). The two layers are cross-linked by carbonate ions (the origin of the carbonates will be discussed later) to form a double layer, and BDC bridges the layers as pillar molecules, resulting in a three-dimensional pillar-layered structure (Figures $2 \mathrm{~b}-2 \mathrm{c}$ ). The $\mu_{5}-\mathrm{CO}_{3}{ }^{2-}$ coordination mode is common in giant cluster synthesis, but is unusual in MOFs. Moreover, we found that the formic acid generated by the decomposition of DMF was also coordinated. Many $\mu$-OH groups are aligned on the KGF-3 pore surfaces, and disordered guest molecules (most likely water) are incorporated in the pores, suggesting that the pores are highly hydrophilic in nature.

However, KGF-3 was difficult to isolate, and its preparation suffered from a poor reproducibility, with different phases frequently obtained even under the same synthesis conditions. In addition, a pure KGF-3 phase was not obtained after 108 experimental trials. To optimize the synthetic conditions, we extracted the dominant factors of the reaction using machinelearning techniques. To predict the conditions under which KGF3 can be obtained more reproducibly, it was necessary to determine whether or not the reaction was successful using PXRD. However, the products are complex mixtures of different phases in many cases; hence, assigning each PXRD pattern to an appropriate phase is challenging. A simple method that excluded arbitrariness was therefore required; hence, we classified the obtained patterns using cluster analysis. ${ }^{[7]}$ All diffraction patterns were automatically analyzed and successfully classified into six categories, in which the main products were KGF-3 (cluster 1), four reported phases (clusters $2^{[8]}, 3^{[9]}, 4^{[10]}$ and $\left.5^{[11]}\right)$, and another unknown phase (cluster 6 ), as shown in Figures $2 \mathrm{e}$ and $\mathrm{S1}$. By examining these results, we revealed that automated classification was consistent with researcher intuition", (a)

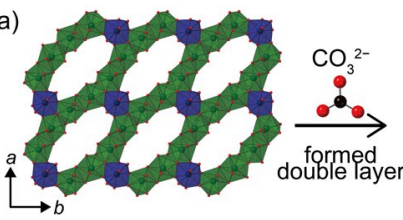

(c)

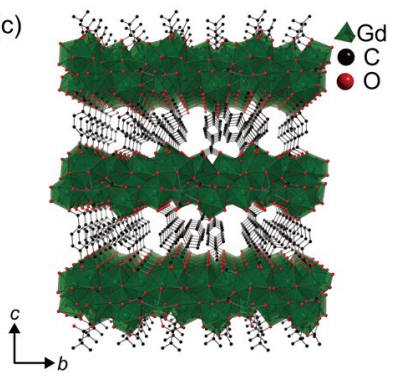

(e)

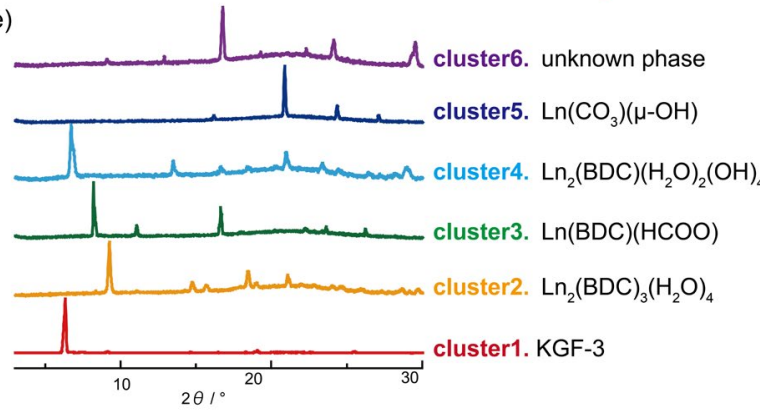

(b)

(d)

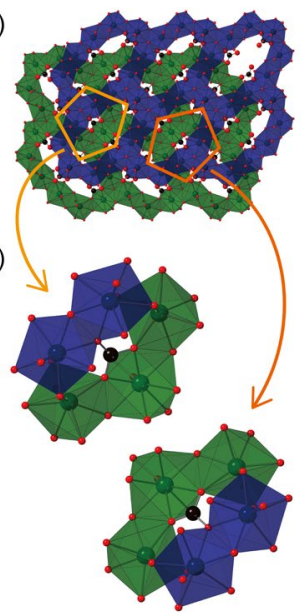

Figure 2. (a) Monolayer with 10 spread-out lanthanide clusters. (b) Double layer connected by carbonate ions. (c) KGF-3(Gd) viewed along the aaxis. $\mathrm{H}$ atoms are omitted for clarity. (d) A pentagonal pocket connected by a carbonate ion. (e) Using cluster analysis to classify the acquired PXRD patterns. 


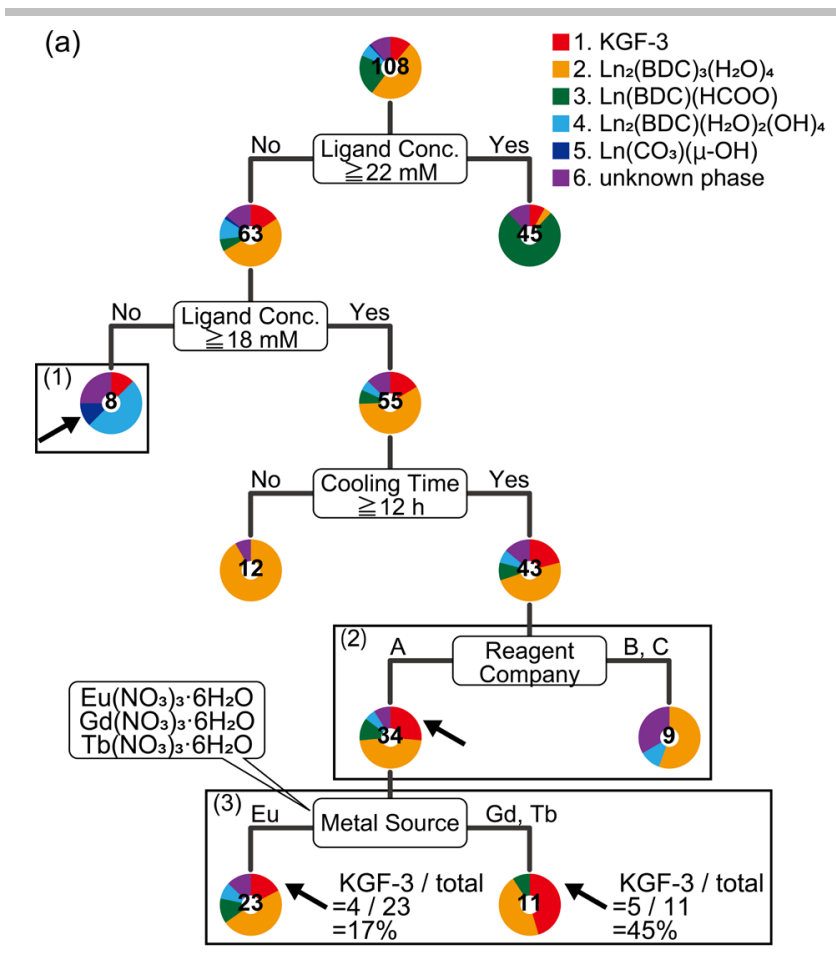

(b)

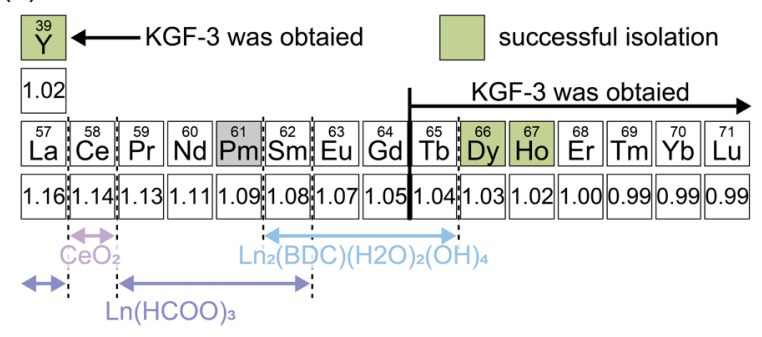

Figure 3. (a) Visualizing the relationships between the experimental conditions and the products, based on decision tree analysis. Ovals show the decision nodes, the pie charts show the product ratios, and the number of experiments is shown in the center of each pie chart. (1), (2), and (3) are branches related to concentration conditions, reagent company, and lanthanide metal, respectively. Branches that are not discussed in the main text have been omitted. The complete version of the decision tree is given in Figure S2. (b) Summary of the KGF-3 synthetic results under Synthetic Conditions 3 . See $\mathrm{S} 7$ for details.

with the exception of only two data points in 108 patterns (see Cluster analysis in $\mathrm{SI}$ ).

We next turned our attention to extracting the dominant factors responsible for the poor reproducibility using decision tree analysis, which is considered to be one of the most interpretable machine-learning techniques.[6a, 6f, 6h] Initially, the experimental data and cluster analysis results were linked together in a text file, after which the data file was analyzed using the decision tree technique, where the objective variables were the crystal phases assigned by cluster analysis of the PXRD patterns, and the explanatory variables were the synthetic parameters (see Decision tree analysis in $\mathrm{SI}$ ). The results presented in Figure $3 \mathrm{a}$ suggest that the most suitable synthetic conditions for the preparation of KGF-3 are as follows: ligand solution concentration, 18-22 mM; cooling time, $>12 \mathrm{~h}$; and metal salt source, company A. The parameters appearing at the branches in the decision tree were also suggested to be important by random forest analysis

(Figure S3). Thus, visualization of the experimental data by decision tree analysis allowed us to understand the synthetic conditions at a glance. The information extracted from the decision tree is summarized as follows. Firstly, it is likely that when the ligand concentration is $<18 \mathrm{mM}$, a product is formed with bridging carbonate ligands (cluster 5) (Figure 3a-(1)), suggesting that carbonate is generated by the decomposition of DMF or through capture from the air. ${ }^{[12]}$ Secondly, the success or failure of the KGF-3 synthesis was determined by the reagent company employed, with the nitrate provided by company $A$ being most suitable (Figure 3a-(2)). Finally, as shown in Figure 3a-(3), the success rate was $17 \%$ on the left branch (Eu) and $45 \%$ on the right branch ( $\mathrm{Gd}$ and $\mathrm{Tb}$ ), suggesting that the metal ion affects the synthetic process. To determine the synthetic conditions that maximize reproducibility, the extracted dominant factors were verified as follows.

In terms of the supply company for the lanthanide nitrate, the reagents purchased from company A were superior (Figure S4), exhibiting a higher purity (99.95\%) compared to those obtained from companies B (99.9\%) and C (99.5\%). The purities guaranteed by the reagent companies were evaluated based only on the metal ion concentration. To estimate the influence of the purity of the metal source, $\mathrm{Tb}\left(\mathrm{NO}_{3}\right)_{3} \cdot 6 \mathrm{H}_{2} \mathrm{O}$ with the highest purity $(99.999 \%)$, i.e., from company D, was also used, which gave an improved success rate compared to that obtained using $\mathrm{Tb}\left(\mathrm{NO}_{3}\right)_{3} \cdot 6 \mathrm{H}_{2} \mathrm{O}$ from company $\mathrm{A}$; the success rate was $20 \%(7$ out of 34 trials) using the Tb source from company A while the success rate increased to be $95 \%$ using that from company D (19 out of 20 trials), as shown in Figure S5. The different purities were evaluated by inductively coupled plasma-mass spectrometry, which revealed that the $\mathrm{Tb}(\mathrm{NO})_{3} \cdot 6 \mathrm{H}_{2} \mathrm{O}$ obtained from company $\mathrm{A}$ contained slightly higher levels of Eu than that from Company $D$ (Table S2). Therefore, KGF-3 synthesis was carried out using lanthanide salts purchased from company $D$ in all of the following experiments.

The results from decision tree analysis also showed that under low concentration conditions, carbonate ions are captured within the structure (cluster 5). Generally, carbonate ions play important roles as anion templates during the formation of polynuclear lanthanide clusters. ${ }^{[2 e, 13]}$ In many cases, carbonate ions are generated by the decomposition of the precursor and/or the uptake of carbon dioxide from the air. Therefore, in the crystal structure of KGF-3, it is likely that the molecules coordinating to five metal ions in a pentagonal pocket (Figure 2d) are carbonate ions. To estimate the effect of these carbonate ions, KGF-3 was synthesized with the addition of sodium carbonate (Synthetic Conditions 2, $\mathrm{SI}$ ). For $\mathrm{Gd}$ and $\mathrm{Tb}$, the formation of the $\mathrm{Ln}_{2}(\mathrm{BDC})_{3}\left(\mathrm{H}_{2} \mathrm{O}\right)_{4}$ impurity (cluster 2) was suppressed with the addition of carbonate ions. In the case of Eu, $\operatorname{Ln}_{2}(B D C)_{3}\left(H_{2} O\right)_{4}$ (cluster 2) was preferentially synthesized, regardless of whether carbonate ions were added or not (Figure S6). These results suggest that the effect imparted by the carbonate ions depends on the lanthanide metal ion.

The decision tree analysis (Figure 3a-(3)) and the response to the addition of carbonate ions strongly suggest that the metal ion affects the probability of successfully synthesizing KGF-3. We therefore synthesized KGF-3(Ln) with various lanthanide metal ions (La-Lu, excluding $\mathrm{Pm}$ ) under optimized Synthetic Conditions 3 and 4 (see SI, also Figures 3b and S7). Based on PXRD and elemental analysis (EA) data, only Dy and Ho provided pure KGF- 
3. The EA results show that no nitrogen atom exists in KGF-3 (Table S4), thereby confirming that all three directional ligands in the crystal structure are carbonate ions (see Elemental analysis in $\mathrm{SI}$ ). The observation that the presence of trace amounts of Eu in the purchased reagent render the experiments more likely to fail coincides with the fact that other crystal phases form with high probabilities using Eu. In many cases, the ionic radius of the lanthanide ion strongly influences the construction of the obtained Ln complex. ${ }^{[14]}$ In fact, KGF-3(Y) was successfully isolated when $\mathrm{Y}^{3+}$, whose ionic radius is close to those of $\mathrm{Dy}^{3+}$ and $\mathrm{Ho}^{3+}$ (Figure

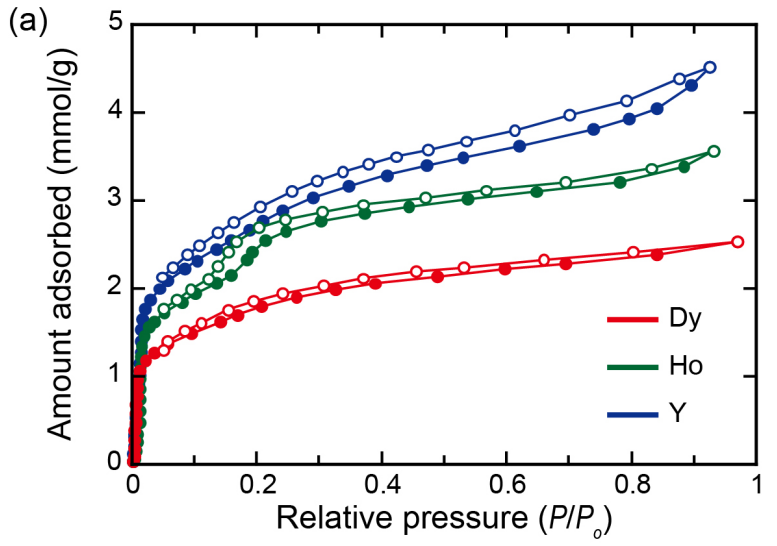

(b)

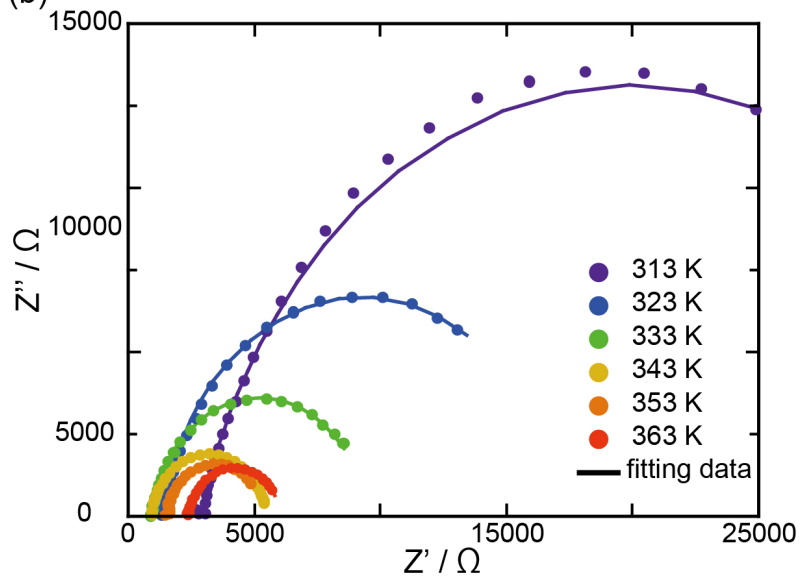

(c)

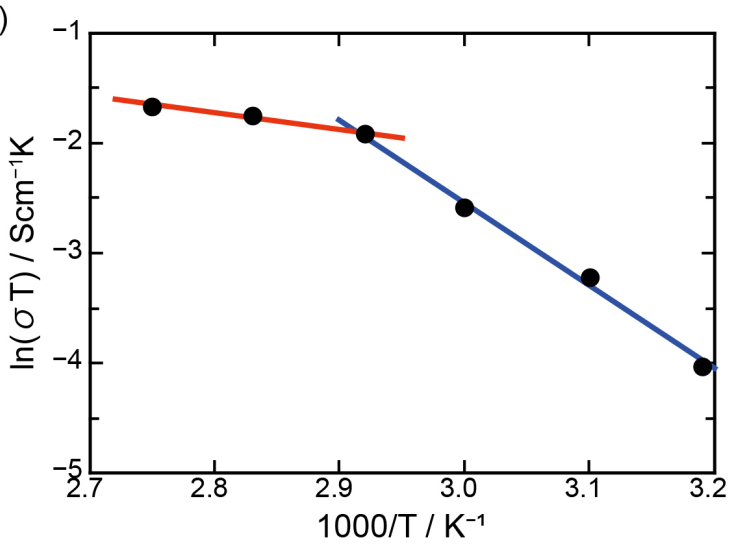

Figure 4. (a) Adsorption and desorption isotherms for $\mathrm{H}_{2} \mathrm{O}$. The solid and open symbols correspond to adsorption and desorption, respectively. (b) Nyquist plots at various temperatures and at $95 \% \mathrm{RH}$ for a pellet sample of KGF-3(Y). (c) Arrhenius plot of the ion conductivity at 95\% RH of KGF$3(\mathrm{Y})$.
$3 b)$, was used, which strongly suggests that the ionic radius of the lanthanide metal is a crucial and dominant factor for KGF-3 formation.

To evaluate the permanent porosity of KGF-3(Dy, Ho, and Y), $\mathrm{N}_{2}$ and $\mathrm{H}_{2} \mathrm{O}$ adsorption isotherms were acquired (Figures $4 a$ and $\mathrm{S} 10$ ), which suggests that KGF-3 adsorbs $\mathrm{H}_{2} \mathrm{O}$ molecules into its pores, whereas $\mathrm{N}_{2}$ is not adsorbed; hence KGF-3 is likely to possess narrow hydrophilic channels whose diameters are too small for nitrogen diffusion at $77 \mathrm{~K}$; this result is consistent with the obtained crystal structure of KGF-3. Although the water molecules are disordered within the pores, they are expected to form a pathway for proton conduction, with the water assembly stabilized by the hydrophilic pore surface (Figure S12). The hydrophilic nature of KGF-3 therefore prompted us to evaluate its proton conductivity. Thus, the alternating current impedances of KGF-3(Dy, Ho, and Y) were measured at $313-363 \mathrm{~K}$ at $95 \%$ relative humidity (Figures $4 \mathrm{~b}$ and $\mathrm{S} 13$ ). KGF-3(Y) showed the highest proton conductivity among the three MOFs, i.e., $5.2 \times 10^{-4}$ $\mathrm{S} \mathrm{cm}^{-1}$ at $363 \mathrm{~K}$, and KGF-3 retained its crystalline nature after the impedance experiments or even after soaking in water (Figures S11 and S13). The conductivity of KGF-3(Y) was observed to increase with increasing temperature due to thermal activation of water molecules. The activation energy for proton conduction was calculated to be $0.65 \mathrm{eV}$ at low temperatures (40$343 \mathrm{~K})$, whereas at high temperatures $(343-363 \mathrm{~K})$, it was 0.14 $\mathrm{eV}$ (Figure 4c), suggesting that changes in the transport mechanism occurs at $\sim 343 \mathrm{~K}^{.15]}$

In summary, we successfully synthesized a series of novel pillar-layered Ln-MOFs with lanthanide double-layer based inorganic subnetworks that we refer to as "KGF-3". Although it was difficult to isolate KGF-3 in our first screening experiments due to the poor synthetic reproducibility, we successfully extracted the dominant factors for KGF-3 synthesis by evaluating both failure and success using machine-learning techniques. The extracted chemical insight suggests that the lanthanide ion affects the synthesis results, and systematic synthesis experiments demonstrated the effect of the ionic radius of the metal ion. This method is a useful tool for preparing new MOFs and related compounds, such as coordination polymers and covalent organic frameworks that suffer from poor synthetic reproducibilities. Through the application of this method, the exploration of novel MOFs and coordination polymers, for which it is challenging to obtain highly crystalline samples, is currently underway, and the results will be presented in due course.

\section{Acknowledgements}

This work was supported by grants from JST PRESTO (Grant No. JPMJPR17NA), and JSPS KAKENHI (Grant Nos. 20H02577, 17K00320, 20H04680, 20H04646). We thank Dr. Satoki Okabayashi, Prof. Koichi Chiba and Dr. Akane Ito (KwanseiGakuin University) for ICP-OES suppor. This work was performed under the Cooperative Research Program of "Network Joint Research Center for Materials and Devices." Prof. Masaharu Tanimizu was supported by a Joint Research Grant for the ICP-MS experiments from the Environmental Isotope Study of the Research Institute for Humanity and Nature.

Keywords: lanthanide $\bullet$ machine learning $\bullet$ metal-organic framework $\cdot$ proton conductivity $\cdot$ solvothermal synthesis 
[1] a) J. Rocha, L. D. Carlos, F. A. Paz, D. Ananias, Chem. Soc. Rev. 2011, 40, 926; b) S. Roy, A. Chakraborty, T. K. Maji, Coord. Chem. Rev. 2014, 273, 139; c) W. P. Lustig, S. Mukherjee, N. D. Rudd, A. V. Desai, J. Li, S. K. Ghosh, Chem. Soc. Rev. 2017, 46, 3242; d) X. Meng, H. N. Wang, S. Y. Song, H. J. Zhang, Chem. Soc. Rev. 2017, 46, 464; e) F. Saraci, V. Quezada-Novoa, P. R. Donnarumma, A. J. Howarth, Chem. Soc. Rev. 2020, 49, 7949; f) P. Cheng, Lanthanide Metal-Organic Frameworks, Springer, 2015.

[2] a) J. B. Peng, X. J. Kong, Q. C. Zhang, M. Orendac, J. Prokleska, Y. P. Ren, L. S. Long, Z. Zheng, L. S. Zheng, J. Am. Chem. Soc. 2014, 136, 17938; b) J. Dong, P. Cui, P. F. Shi, P. Cheng, B. Zhao, J. Am. Chem. Soc. 2015, 137, 15988; c) K. Wang, Z. L. Chen, H. H. Zou, K. Hu, H. Y. Li, Z. Zhang, W. Y. Sun, F. P. Liang, Chem. Commun. 2016, 52, 8297; d) J. Wang, M. Feng, M. N. Akhtar, M. L. Tong, Coord. Chem. Rev. 2019, 387, 129; e) X.-Y. Zheng, J. Xie, X.-J. Kong, L.-S. Long, L.-S. Zheng, Coord. Chem. Rev. 2019, 378, 222.

[3] a) G. Ferey, Chem. Soc. Rev. 2008, 37, 191; b) T. Devic, C. Serre, Chem. Soc. Rev. 2014, 43, 6097; c) H. N. Miras, L. Vila-Nadal, L. Cronin, Chem. Soc. Rev. 2014, 43, 5679.

[4] a) N. Stock, S. Biswas, Chem. Rev. 2012, 112, 933; b) M. J. Van Vleet, T. Weng, X. Li, J. R. Schmidt, Chem. Rev. 2018, 118, 3681; c) X. Gong, H. Noh, N. C. Gianneschi, O. K. Farha, J. Am. Chem. Soc. 2019, 141, 6146; d) B. Karadeniz, D. Zilic, I. Huskic, L. S. Germann, A. M. Fidelli, S. Muratovic, I. Loncaric, M. Etter, R. E. Dinnebier, D. Barisic, N. Cindro, T. Islamoglu, O. K. Farha, T. Friscic, K. Uzarevic, J. Am. Chem. Soc. 2019, 141, 19214.

[5] a) X. Yang, Y. Wang, R. Byrne, G. Schneider, S. Yang, Chem. Rev. 2019, 119, 10520; b) C. W. Coley, N. S. Eyke, K. F. Jensen, Angew. Chem. Int. Ed. 2020, 59, 22858; c) S. M. Moosavi, K. M. Jablonka, B. Smit, J. Am. Chem. Soc. 2020, 142, 20273.

[6] a) P. Raccuglia, K. C. Elbert, P. D. Adler, C. Falk, M. B. Wenny, A. Mollo, M. Zeller, S. A. Friedler, J. Schrier, A. J. Norquist, Nature 2016, 533, 73; b) V. Duros, J. Grizou, W. Xuan, Z. Hosni, D. L. Long, H. N. Miras, L. Cronin, Angew. Chem. Int. Ed. 2017, 56, 10815; c) R. L. Greenaway, V. Santolini, M. J. Bennison, B. M. Alston, C. J. Pugh, M. A. Little,
M. Miklitz, E. G. B. Eden-Rump, R. Clowes, A. Shakil, H. J. Cuthbertson, H. Armstrong, M. E. Briggs, K. E. Jelfs, A. I. Cooper, Nat. Commun. 2018, 9, 2849; d) V. Duros, J. Grizou, A. Sharma, S. H. M. Mehr, A. Bubliauskas, P. Frei, H. N. Miras, L. Cronin, J. Chem. Inf. Model. 2019, 59, 2664; e) S. M. Moosavi, A. Chidambaram, L. Talirz, M. Haranczyk, K. C. Stylianou, B. Smit, Nat. Commun. 2019, 10, 539; f) K Muraoka, Y. Sada, D. Miyazaki, W. Chaikittisilp, T. Okubo, Nat. Commun. 2019, 10, 4459; g) K. M. Jablonka, D. Ongari, S. M. Moosavi, B. Smit, Chem. Rev. 2020, 120, 8066; h) Y. Xie, C. Zhang, X. Hu, C. Zhang, S. P. Kelley, J. L. Atwood, J. Lin, J. Am. Chem. Soc. 2020, 142, 1475.

[7] B. M. Butler, A. M. Sila, K. D. Shepherd, M. Nyambura, C. J. Gilmore, N. Kourkoumelis, S. Hillier, Geoderma 2019, 337, 413.

[8] T. M. Reineke, M. Eddaoudi, M. Fehr, D. Kelley, O. M. Yaghi, . Am. Chem. Soc. 1999, 121, 1651.

[9] G. Huang, P. Yang, N. Wang, J.-Z. Wu, Y. Yu, Inorg. Chim. Acta 2012, 384, 333

[10] C. Serre, F. Millange, J. Marrot, G. Ferey, Chem. Mater. 2002, 14, 2409.

[11] H. Tian, L. Zhao, Y. N. Guo, Y. Guo, J. Tang, Z. Liu, Chem. Commun. 2012, 48, 708 .

[12] a) B. Zhang, X. Zheng, H. Su, Y. Zhu, C. Du, M. Song, Dalton Trans. 2013, 42, 8571; b) H.-M. Chen, W.-M. Wang, X.-Q. Li, X.-Y. Chu, Y.-Y. Nie, Z. Liu, S.-X. Huang, H.-Y. Shen, J.-Z. Cui, H.-L. Gao, Inorg. Chem. Front. 2018, 5, 394.

[13] a) X. Y. Zheng, Y. H. Jiang, G. L. Zhuang, D. P. Liu, H. G. Liao, X. J. Kong, L. S. Long, L. S. Zheng, J. Am. Chem. Soc. 2017, 139, 18178; b) Y. Zhou, X. Y. Zheng, J. Cai, Z. F. Hong, Z. H. Yan, X. J. Kong, Y. P. Ren, L. S. Long, L. S. Zheng, Inorg. Chem. 2017, 56, 2037; c) X. Y. Li, H. F. Su, Q. W. Li, R. Feng, H. Y. Bai, H. Y. Chen, J. Xu, X. H. Bu, Angew. Chem. Int. Ed. 2019, 58, 10184.

[14] a) H. P. Xiao, J. Zhou, R. Q. Zhao, W. B. Zhang, Y. Huang, Dalton Trans. 2015, 44,6032; b) M. Ellart, F. Blanchard, M. Rivenet, F. Abraham, Inorg. Chem. 2020, 59, 491.

[15] a) P. Ramaswamy, N. E. Wong, G. K. Shimizu, Chem. Soc. Rev. 2014, 43, 5913; b) D. W. Lim, H. Kitagawa, Chem. Rev. 2020, $120,8416$. 